\section{PHYSIOLOGY OF DISEASE RESISTANCE IN PLANTS}

\begin{abstract}
$\mathrm{A}^{\mathrm{T}}$ T the Royal Society symposium held on December 11 on the "Physiology of Resistance to Disease in Plants", three papers, by Prof. W. Brown of the Imperial College of Science and Technology, Prof. F. T. Brooks of the University of Cambridge and Dr. F. C. Bawden of the Rothamsted Experimental Station, respectively, were read. Unfortunately, there was no time for a general discussion at the end of the papers.

Prof. W. Brown claimed that physiological analysis of the host-parasite relationship is most readily attempted in the case of the facultative parasite. The latter can be cultivated in vitro and its metabolism, therefore, studied up to the limits set by available experimental techniques. One can thus obtain some idea of the means by which fungi and bacteria attack plants-the so-called 'offensive mechanism' of the parasite - and conversely of the resistance which plants are able to oppose to such attack. The establishment of parasitism involves the carrying out of a series of operations which will be discussed in the following sequence: (1) pre-penetration phase, (2) the act of penetration, and (3) postpenetration phase.

In the pre-penetration phase the first requisite is that spore germination should take place. The more vigorous the germination the more likely is penetration to occur. Moist conditions, that is, presence of free water or of a sufficiently high relative humidity, are essential, and these must persist for a time sufficient to allow of germination and entrance into the host, otherwise death of the germ-tubes by desiccation would result. Meteorological conditions largely determine whether a particular plant disease can establish itself or whether alternatively it reaches epidemic proportions. Even under wet-weather conditions, some plants are protected from invasion by possessing an unwettable type of surface, so that one of the preliminary conditions for infection is not satisfied. This is an example of 'disease escape', the plant not being attacked even though it may be inherently susceptible.
\end{abstract}

Granted suitable environmental conditions, the germinative capacity of the spores of any fungus varies with their age and with the conditions of culture under which they were grown. A factor of importance at this stage is the passive exosmosis of solutes from the underlying host cells, and these affect the amount and vigour of spore germination according to their quantity and quality. The presence of other fungi or of bacteria in the inoculum has in general a depressing effect on germination and therefore on the establishment of parasitism. This is an example of microbiological antagonism which is more prominently shown with certain root-invading fungi.

The stimulus to penetration was at one time considered to be of chemotropic nature, but that view has been largely discarded. With fungi which penetrate directly through the cuticle, the stimulus is one of mechanical contact, the germ tubes becoming fixed to the surface of the host by formation of an appressorium (attachment organ), at which point they attempt to penetrate. The mechanism of penetration is also physical, the fungus putting an extremely fine outgrowth through the cuticle. The evidence on this point is partly cytological and partly that no cuticle-dissolving substance is known to be produced by fungi. The mechanical properties of cuticularized epidermis and the intrinsic penetrative capacities of fungi are the determining factors at this stage. A part of cuticular resistance depends upon the hydrostatic backing of the underlying epidermal cells. By these means one can explain the penetration of young or old leaves when the leaf is not penetrable in its mature, fully active condition.

Cuticular resistance is the only means of defence of some plants against specific parasites (wound parasites). Frequently the cuticular barrier is circumvented by invasion through stomata or other natural opening, or by way of parts (for example, the stigmas of flowers) where the cuticle is poorly developed. Once inside the plant, parasites of the facultative type disorganise the host tissue and live on the dead remains. The chief substance concerned in this process is pectinase enzyme. Resistance may take the form of retardation or inhibition of growth of the invader or interference with its offensive mechanism. Thus acidity of cell-sap, or presence of tannins, oils, glucosides, etc., may prevent growth of a potential parasite. A similar effect is obtained when the metabolism of the latter leads to the formation of substances ('staling substances') which by and by inhibit growth; hence the formation of lesions of limited size.

The reaction of the host to the presence of the parasite may be to lay down mechanical barriers (gum, cork) which seal off the lesion. Chemical responses, for example, the localized formation of phenolic compounds, may occur; but it is not clear how far these are effective. It is very doubtful if there is in plant pathology (apart from some viruses) an antigen-antibody type of reaction comparable to what is known in animal diseases.

A more general type of resistance is seen where, in the absence of any obvious anti-fungal property of the plant sap, the plant in its normal condition shows resistance to a fungus which appears to have the necessary offensive mechanism. Slight modifications of the host, as produced by raising its water-content or by previous incubation at a warm temperature, may swing resistance over to susceptibility. Other components of this problem are the differential effects of nutrient upon the capacity of fungi to produce pectinase, and differences in detailed properties of pectinase as derived from various fungi. The continuance or otherwise of attack appears to rest upon the functioning of an intricate enzymic process, the details of which have not been worked out as yet.

Finally, there is a kind of resistance which depends upon insensitiveness of the plant tissue to the enzymic secretion of the fungus. As the primary enzymic effect is hydrolysis of the cell walls, the factor which confers immunity in this case is the chemical com. position of the cell wall.

Prof. F. T. Brooks pointed out that the fundamental difficulty met with in studying the physiology of obligate parasites is that the fungus cannot be grown on culture media and thus has to be studied in relation to the host itself. The relationship of host and parasite is bound up with subtle protoplasmic properties of the two organisms, though even here there is some evidence that structural properties of the host confer a measure of resistance; for example, the presence of a greater amount of sclerenchyma in certain wheats, the thickness of the mature cuticle in the barberry leaf which resists penetration by the germinating sporidia, etc. Resistance of cereals to rust attack has long been known to rest upon the 
'hypersensitive' effect, invasion by the fungus leading to localized necrosis of the host cells, so that further advance of the parasite is stopped. With the fully susceptible host the rust establishes a kind of common life with the invaded tissue and there is no evidence of mutual antagonism. Intergrades between the two types of reaction occur.

One can do little more than speculate as to the physiological nature of these reactions. It has been suggested that hypersensitiveness arises by the excretion from the fungus of a toxin and that the fungus eventually dies from starvation or more probably through the agency of lethal substances excreted by the host. No satisfactory explanation in biochemical terms is as yet available of the difference between susceptible and resistant host varieties.

The hypersensitive reaction is affected by changes in the environment; for example, by nutritional alterations of the host as brought about by varied illumination or by differential manuring, and even by the presence of another parasite in the tissue. Thus under certain conditions a susceptible host becomes resistant and vice versa. Frequently suscep. tibility in seedling cereal plants gives way to resistance as the plants grow older ('mature plant resistance'). This effect is of great practical importance, but its physiological meaning is unknown.

Resistance to attack by mildews first becomes evident at the stage where a sub-cuticular papilla is formed. Whereas in a susceptible host a haustorium emerges from the papilla to invade the underlying epidermal cell, such emergence does not occur where there is resistance. The host cell is undamaged, though a hypersensitive effect has also been described. A similar range of behaviours is seen in connexion with the invasion of potato varieties by the wart disease fungus, though here the striking response is the large development of gall tissue when the variety is susceptible.

With certain facultative parasites there are defensive mechanisms in resistant hosts which, while not preventing initial invasion, preclude the fungus from spreading extensively in the tissues. Such is the gum barrier produced from wood carbohydrates when a resistant variety of plum is invaded by the silverleaf fungus. A susceptible variety produces no such protective barrier, though under conditions of enhanced metabolism it may do so to some extent. Resistance to the root-invading fungus, Armillaria mellea, also appears to be due in part to the gumming reaction, though here cork barriers are developed in addition. The functioning of cork barriers is well seen in varieties of apple trees resistant to canker; in this case the effectiveness of the barrier is largely dependent upon the state of metabolism of the host, so that under some conditions a normally resistant variety becomes susceptible. The well-known 'shot-hole' appearance in the leaves of many plants is often due to the limitation of the lesion by a cork barrier.

Resistance to wilt-producing fungi appears to arise in a number of ways. With flax-wilt, resistance is seated in the cortex of the roots, so that the fungus is shut off from reaching the vascular bundles. In hop varieties resistant to wilt, the fungus enters the vessels, where it grows much as in a susceptible variety, but no harm ensues. It has always been considered that the wilting effect is due to the formation by the fungus of a toxin, and recently the toxin of tomato wilt has been identified as a polypeptide of known composition. Presumably the resistance of certain hop varieties to wilt is due to the non-elabora- tion of a toxin, or to insensitiveness of these varieties to the toxin produced. In some cases resistance of plants to wilt appears to be a property of the root only, in others of the whole plant.

Among the few examples in which resistance to fungal invasion is associated with particular substances of known composition, the best known is that of coloured types of onion against certain parasites. In contrast to white varieties of onion, which are susceptible, coloured ones owe their resistance to the presence in their outer scales of catechol and protocatechuic acid.

Resistance is rarely absolute under all conditions. The precise result obtained depends to some extent on the amount of inoculum applied and on environmental factors as affecting both host and parasite. The latter has already been referred to in connexion with resistance of cereals to rusts. A notable example of the effect of temperature on disease response is seen with the scab disease (Fusarium) of wheat and maize. Wheat seedlings are susceptible at the higher, maize seedlings at the lower temperature ranges, and in both cases it has been shown that susceptibility is correlated with a pectic type of cell-wall structure, the temperature under which the plants were grown being the determining factor for this.

Dr. F. C. Bawden dealt mainly with viruses. These are unique pathogens, for they are both wound parasites and obligate parasites. All uninjured plants are immune from all viruses, and most viruses have a narrow host range. Thus immunity is the common state, susceptibility is exceptional. Nothing is known of the conditions conferring natural immunity. The growth requirements of viruses are unknown, and though plant extracts often contain virus inhibitors it is uncertain whether these substances function as such in vivo. One is, therefore, limited to considering variations in degree and type of resistance shown by susceptible plants.

Viruses rely for transmission on vectors, chiefly leaf-sucking insects, and usually produce systemic infections. Possibly because of their systemic nature, virus diseases provide the only authentic examples of acquired resistance in plant pathology. There are two types, both simulating conditions well known with animal diseases. In the first type, infection leads to severe initial symptoms, from which the plants recover, and then resist effects of further inoculation. The recovery is not because of antibody formation, and the virus is present all the time in the resistant tissues. The second type of acquired resistance simulates the process of vaccination in animals : protection against a severe strain of a virus is given by previous infection with a mild strain. Again there is no antibody formed. Protection is based on mutual antagonism between the two viruses. It is suggested that there are only a limited number of sites of virus multiplication in a cell, and if these are occupied by one strain another cannot become established. The resistance is acquired only against related strains, and unrelated viruses may act synergistically, giving together severe symptoms when each alone gives only mild.

Virulence is not an intrinsic property of a virus strain but a reflexion of a host-parasite interaction, and is not correlated with the concentration of virus reached within the plant. Strains of potato virus $X$, for example, may cause severe diseases in some potato varieties and not in others. Hypersensitiveness, similar to that of cereals to rust fungi, affords a common form of resistance. Sometimes, however, 
there is a systemic hypersensitiveness in which the whole plant is invaded and killed. This is the extreme of susceptibility, but confers resistance by eliminating the virus from the stock as a whole. Varieties of potato which react to virus $X$ in this way are rarely found infected in the field, whereas other varieties are almost universally so.

Short of hypersensitiveness, intermediate degrees of tolerance occur, down to the condition of 'carrier' varieties which show no symptoms though they may contain a high concentration of virus. The degree of tolerance is somewhat modified by environmental conditions.

With equal chance of infection, a greater incidence of disease occurs in some varieties than in others. This has been demonstrated both in field and controlled experiments. The inference is that different amounts of virus are needed to cause infection in different hosts. Though the evidence from dilution curves is that infection is caused by single virus particles, it seems that different numbers must be introduced into different cells to ensure that one becomes established.

With some viruses infection occurs only when the particles are introduced directly into the phloem; more often introduction into ordinary parenchyma cells is sufficient. The method of inoculation can affect susceptibility, infection taking place more readily when the inoculum includes a suitable abrasive. The severity of the symptoms shown may also be increased by this means.

An effect comparable to the 'mature plant' resist. ance of cereals to rust fungi is often seen, ease of infection and the severity of symptoms diminishing as the plant grows older. The cultural conditions of the host also influence resistance; thus some viruses are more readily transmitted in winter than in summer. The controlling factor appears to be light, and susceptibility to some viruses is enhanced by keeping the plants in darkness for 24 hours before inoculation. It is suggested that the presence of photosynthetic products interferes with the establishment of virus particles at sites of multiplication. The conditions of mineral nutrition are also important, phosphorus being most effective in increasing susceptibility, though quantitative comparisons are rendered difficult by unequal growth of the plant under different systems of manuring. Increased size of the plant itself tends to favour infection, and conversely small size gives plants a disease-escape mechanism which may simulate resistance in the field. Suscep. tible plants may also escape infection because they are unattractive to vectors or grow in situations which reduce the chance of visits by the appropriate insects.

\section{CHEMISTRY OF VISUAL PROCESSES}

\section{By S. BALL, F. D. COLLINS, Prof. R. A. MORTON AND}

\section{DR. A. L. STUBBS}

\section{Biochemistry Department, University of Liverpool}

CINCE rhodopsin (visual purple) was first described $S$ in 1876 by $\mathrm{F}$. Boll ${ }^{1}$, much evidence concerning its chemical nature and physiological properties has accumulated. Relatively few attempts have been made, however, to devise theories embracing all the facts ; the contributions of G. Wald ${ }^{2}, R$. J. Lythgoe and his colleagues ${ }^{3}$ and R. Granit and his co-workers ${ }^{4}$ being outstanding. The objects of the present article are to describe experiments which seem to reconcile divergent findings, as between Wald and Lythgoe, and to provide a basis for discussing in chemical terms Granit's discoveries, concerning the electrophysiology of retinal elements.

It is necessary first to elucidate the decomposition of rhodopsin extracts. Wald ${ }^{2}$ stated that retinen $\theta_{1}$, later shown by Morton and Goodwin ${ }^{5}$ to be vitamin A aldehyde, was the final product of bleaching. He was able to extract retinene from retinas freshly bleached by light, or from bleached solutions of rhodopsin, by using as solvent a mixture of acetone, ethanol and light petroleum. The retinene was identified by its ultra-violet absorption maximum 380-385 $\mathrm{m} \mu$ in chloroform and its colour test maximum (664 $\mathrm{m} \mu$ ) with the antimony trichloride reagent. When a solution of rhodopsin in the aqueous digitonin used to detach the pigment from the retina was acidified before bleaching, the irradiated solution exhibited an absorption maximum at $443 \mathrm{~m} \mu$ which afterwards disappeared, the final solution having the same general absorption on the long-wave side of $400 \mathrm{~m} \mu$ as neutral or alkaline solutions. At that time Wald was not able to follow the absorption curves on the short-wave side. It seems clear, how. ever, that he regarded retinene ${ }_{1}$ as the sole and final product of bleaching rhodopsin in vitro.

Lythgoe and his co-workers were also restricted to wave-lengths exceeding about $395 \mathrm{~m} \mu$ in recording absorption spectra. They observed that in acidified aqueous digitonin rhodopsin gave on irradiation 'indicator yellow' with $\lambda_{\max } 444 \mathrm{~m} \mu$. In neutral or alkaline solution no maxima (at wave-lengths greater than $395 \mathrm{~m} \mu$ ) were observed. Lythgoe concluded that indicator yellow was the final product of bleaching rhodopsin; he suggested that it was a conjugated protein with $\lambda_{\max } .444 \mathrm{~m} \mu$ shown only in acid.

A. C. Krause $^{6}$ obtained a fraction from bovine retinas which exhibited $\lambda_{\max } 444 \mathrm{~m} \mu$ in acidified ethanol and $360 \mathrm{~m} \mu$ when the solution was made alkaline. Krause obtained his materials from freshly bleached retinas rather than from rhodopsin solutions, and his findings are not strictly comparable.

The work carried out in our laboratory confirms and extends the observations made by both Wald and Lythgoe. Thus in neutral solutions rhodopsin bleaches to a substance exhibiting $\lambda_{\max } 380 \mathrm{~m} \mu$ and on which extraction by means of an ethanol-light petroleum mixture yields retinene ${ }_{1}\left(\gamma_{\max } .385 \mathrm{~m} \mu\right.$ in chloroform, $664 \mathrm{~m} \mu$ with the Carr-Price reagent). In acid solution, bleached rhodopsin shows $\lambda_{\max } .440 \mathrm{~m} \mu$, and in alkaline solution $\lambda_{\max }$ c. $360 \mathrm{~m} \mu$. In neither case is any absorbing material extracted by means of ethanol-petroleum mixture provided the $p H$ is sufficiently different from 7. The substance giving rise in acid to the $440 \mathrm{~m} \mu$ maximum is unstable even in the dark, and the final product is devoid of selective absorption. No retinene $\theta_{1}$ can be extracted at that stage even after neutralization.

Further experiments have been carried out using pure retinen $\theta_{1}$ obtained $^{7}$ by the oxidation of vitamin A. When dissolved in 1 per cent aqueous digitonin, pure retinene shows $\lambda_{\text {max }} .380 \mathrm{~m} \mu$, and the spectrum is unaffected by changing the $p H$ of the medium. If, however, retinene ${ }_{1}$ dissolved in aqueous alcohol or 1 per cent digitonin is mixed with one of the following: (a) an aqueous extract of bleached retinas, 